\title{
ARTICLE
}

Clinical Study

\section{Phase 2 study of NAB-paclitaxel in SensiTivE and refractory relapsed small cell lung cancer (SCLC) (NABSTER TRIAL)}

\author{
Francesco Gelsomino ${ }^{1}$, Marcello Tiseo $\mathbb{D}^{2}$, Fausto Barbieri ${ }^{3}$, Ferdinando Riccardi ${ }^{4}$, Luigi Cavanna ${ }^{5}$, Antonio Frassoldati ${ }^{6}$, \\ Angelo Delmonte ${ }^{7}$, Lucia Longo ${ }^{8}$, Claudio Dazzi ${ }^{9}$, Saverio Cinieri ${ }^{10}$, Ida Colantonio ${ }^{11}$, Francesca Sperandi $^{1}$, Giuseppe Lamberti ${ }^{1}$, \\ Stefano Brocchi ${ }^{12}$, Lorenzo Tofani ${ }^{13}$, Luca Boni ${ }^{13}$ and Andrea Ardizzoni ${ }^{1}$
}

BACKGROUND: Despite sensitivity to first-line chemotherapy, most small-cell lung cancer (SCLC) patients relapse. In this setting, topotecan demonstrated modest activity with significant toxicity. Paclitaxel was also active. This study was designed to evaluate activity and safety of nab-paclitaxel in relapsed SCLC.

METHODS: In this multicentre prospective Phase 2 trial, patients with refractory or sensitive SCLC progressed to first-line platinumbased chemotherapy received nab-paclitaxel $100 \mathrm{mg} / \mathrm{smq}$ on days $1,8,15$ every 4 weeks up to six cycles, progressive disease or intolerable toxicity. Primary endpoint was investigator-assessed objective tumour response. Secondary endpoints were toxicity, progression-free survival (PFS) and overall survival (OS).

RESULTS: Of the 68 patients treated, partial response was $8 \%$ in the refractory cohort and $14 \%$ in the sensitive cohort. Most common toxicities of any grade were fatigue (54\%), anaemia (38\%), neutropenia (29\%), leukopenia (26\%) and diarrhoea (21\%). Median PFS was similar in both refractory (1.8 months) and sensitive cohorts (1.9 months), while median OS was longer in sensitive one (6.6 versus 3.6 months).

CONCLUSIONS: Although nab-paclitaxel has shown some modest anti-tumour activity in relapsed SCLC, associated with a favourable toxicity profile, the primary end-point of the study was not met.

CLINICAL TRIAL REGISTRATION: Clinical Trial registration number is ClinicalTrials.gov Identifier: NCT03219762.

British Journal of Cancer (2020) 123:26-32; https://doi.org/10.1038/s41416-020-0845-3

\section{BACKGROUND}

Small cell lung cancer (SCLC) is one of the most aggressive tumours and accounts for $\sim 13-15 \%$ of all lung cancers. ${ }^{1}$ Most patients with SCLC have extensive-disease (ED-SCLC) at the time of diagnosis, with a median overall survival (OS) of 8-12 months. ${ }^{2}$

In the last 30 years, platinum-based chemotherapy has been the standard of care in first-line setting, providing an objective response rate (ORR) of $70-80 \%$. Unfortunately, despite high sensitivity to first-line chemotherapy, most SCLC patients eventually develop disease progression. ${ }^{3}$ At relapse, efficacy of secondline treatment is modest and highly influenced by the type and duration of response to prior chemotherapy. ${ }^{4}$ Topotecan, the only approved and marketed drug in Europe specifically for the treatment of relapsed SCLC, showed anti-tumour activity (7\% and $21.7 \%)^{5,6}$ and a significant improvement in overall survival (OS) over best supportive care ( 25.9 weeks versus 13.9 weeks, $p=$ $0.0104))^{5,6}$ Nevertheless, it had similar activity (24.3\% versus $\left.18.3 \%\right)$ and efficacy (median OS: 25.0 weeks versus 24.7 weeks) to CAV combination chemotherapy.
However, the anti-tumour activity of topotecan is modest and transient and its use is outweighed by its poor compliance and inconvenient schedule. ${ }^{8}$ Therefore, there is a clinical need for more effective and better tolerated treatments.

Paclitaxel has also shown activity in the treatment of SCLC, both alone and in combination with carboplatin, even in refractory relapsed disease. ${ }^{9-11}$ Notably, the use of paclitaxel is encumbered with a significant risk of severe hypersensitivity reactions and cumulative peripheral neurotoxicity that can limit its use.

Nanoparticles Albumin-Bound (Nab)-paclitaxel (Abraxane ${ }^{\circledR}$; Celgene, Summit, New Jersey) is a new solvent-free formulation of paclitaxel made through high-pressure homogenisation of paclitaxel in presence of serum albumin. In comparison to solvent-based paclitaxel, this formulation, demonstrating a better tumour penetration in preclinical studies, allows reductions in reconstitution volume, infusion time, risk of hypersensitivity reactions, incidence of neutropenia and time needed to recover from peripheral neuropathy. ${ }^{12-14}$

\footnotetext{
${ }^{1}$ Medical Oncology Unit, Policlinico Sant'Orsola-Malpighi, Bologna, Italy; ${ }^{2}$ Medical Oncology Unit, Azienda Ospedaliero-Universitaria of Parma, Parma, Italy; ${ }^{3}$ Medical Oncology

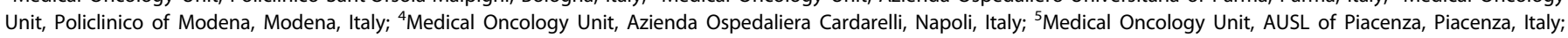

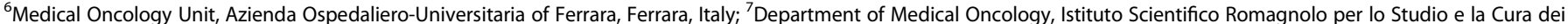

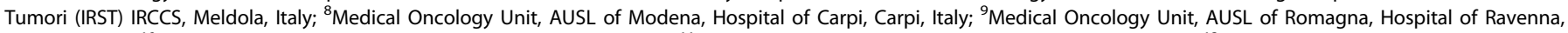

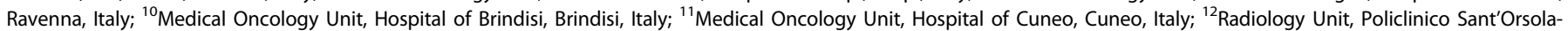
Malpighi, Bologna, Italy and ${ }^{13}$ Clinical Trial Center, Istituto Toscano Tumori, Azienda Ospedaliero-Universitaria Careggi, Firenze, Italy Correspondence: Marcello Tiseo (mtiseo@ao.pr.it)
}

Received: 16 July 2019 Revised: 14 March 2020 Accepted: 1 April 2020

Published online: 29 April 2020 
Nab-paclitaxel is currently approved both as single-agent, for the treatment of metastatic breast cancer, ${ }^{15}$ and as combined therapy with gemcitabine or carboplatin in first-line setting, for the treatment of advanced pancreatic adenocarcinoma ${ }^{16}$ or advanced non-small cell lung cancer (NSCLC), ${ }^{17}$ respectively. Three Asian retrospective analyses conducted in relapsed SCLC patients showed some anti-cancer activity of nab-paclitaxel. ${ }^{18-20}$ Since nab-paclitaxel has not been prospectively studied in relapsed SCLC yet, we designed this open-label, prospective Phase 2 trial with the aim to assess its activity and safety in patients with both refractory and sensitive disease.

\section{METHODS}

Study design and participants

Nabster was a prospective, open-label, multicentre, Phase 2 trial evaluating the activity and safety of nab-paclitaxel in SCLC patients who relapsed during or after first-line platinum-based chemotherapy. Patients were prospectively classified according to treatment free interval (TFI), i.e. the interval from the last chemotherapy administration during first-line chemotherapy and the occurrence of progressive disease, as refractory (TFI $<60$ days) or sensitive (TFI $\geq 60$ days). ${ }^{4}$

Patients aged 18 years or older were eligible for study participation if they had a histological or cytological confirmed diagnosis of SCLC, large cell neuroendocrine carcinoma (LCNEC) or undifferentiated neuroendocrine carcinoma of the lung, according to World Health Organization (WHO) classification 2015, adequate liver, renal and bone marrow functions, measurable disease per Response Evaluation Criteria in Solid Tumors (RECIST) v1.1, ${ }^{22}$ documented radiological evidence of disease progression during or after platinum/etoposide chemotherapy, Eastern Cooperative Oncology Group (ECOG) performance status (PS) 0 to 1. In addition, patients with treated, asymptomatic and stable brain metastases were allowed to be enrolled into the study.

The study protocol was approved by each local institutional ethics committee and conducted in accordance with the $\mathrm{ICH}$ Harmonized Tripartite Guidelines for Good Clinical Practice and the Declaration of Helsinki. Written informed consent was obtained from all participants.

The study was sponsored by Gruppo Oncologico Italiano di Ricerca Clinica (GOIRC) and partially supported by Celgene that provided investigational medicinal product and a restricted grant for the management of study procedures. The trial was registered at ClinicalTrials.gov (number NCT03219762) and assigned its Eudract number (2016-000408-27).

\section{Procedures}

Eligible patients received weekly intravenous administration of nab-paclitaxel $100 \mathrm{mg} / \mathrm{smq}$ on days 1, 8, 15 of a 28-days cycle until a maximum of six cycles, progressive disease or unacceptable toxicity. Treatment could be continued beyond the 6th cycle in patients with confirmed and prolonged objective response, clinical benefit and good tolerance to study drug. Dose reductions and delays were permitted as per-protocol definitions (Study protocol is available in S.1, Supplemental Data). At screening, disease assessment included a computed tomography (CT) scan of the thorax and upper and lower abdomen with contrast. A brain CT or magnetic resonance imaging (MRI) scan had to be performed only if previously abnormal or clinically indicated.

Tumour response was assessed with computed tomography (CT) scan every 8 weeks ( \pm 7 days), according to RECIST criteria v.1.1, and at least 4 weeks after the first observation of a complete or partial response. Furthermore, brain $\mathrm{CT}$ scans had to be repeated if initially abnormal or to be performed if clinically indicated. Patients who discontinued nab-paclitaxel without evidence of progressive disease, continued to be evaluated for disease status every 8 weeks, unless they started new anti-cancer therapy. Complete response (CR) was defined as the complete disappearance of all target lesions and all non-target lesions, if present. Partial response (PR) was defined as at least a $30 \%$ decrease in the sum of diameters of target lesions, taking as reference the baseline sum diameters. Progressive disease (PD) was defined as at least a $20 \%$ increase in the sum of diameters of target lesions, taking as reference the smallest sum on study. The appearance of one or more new lesions and/or unequivocal progression of pre-existing non-target lesions were also considered criteria defining disease progression. Laboratory testing was performed before each study drug administration.

\section{Outcomes}

The primary endpoint was objective tumour response. Tumour response was evaluated according to standard RECIST v.1.1 and based on Investigator's assessment. Data were reported as percentage of $C R, P R$, stable disease (SD) and PD. Patients with no tumour assessment after baseline were classified as nonresponders. Furthermore, to ensure consistency of tumour response measurements among Centres, CT scans performed for all evaluable patients at baseline and during study treatment could be reviewed by a blinded independent radiological committee (BIRC).

Secondary endpoints were toxicity, progression-free survival (PFS) and overall survival (OS). The assessment of safety was based mainly on the frequency of adverse events; toxicity was measured according to $\mathrm{NCl}$ Common Toxicity Criteria Adverse Events ( $\mathrm{NCl}-$ CTCAE), version 4.03 .

PFS was defined as the time from the date of patient's registration to the date of the evidence of progressive disease, death due to any cause, or the last date the patient was known to be progression-free or alive. OS was calculated from the date of patient's registration to the date of death from any cause or the last date the patient was known to be alive.

Statistical design

The aim of this study was to evaluate if nab-paclitaxel objective tumour response rate in each of the two cohorts, sensitive and refractory relapsed SCLC, was sufficient to justify further investigation of the drug in these patients.

In refractory disease, an objective response rate (ORR) $\leq 5 \%$ would not have been considered of further interest. According to the Fleming's single stage design, based on our hypothesis that experimental treatment could guarantee an ORR $\geq 20 \%$ (for a $5 \%$ significance level and $80 \%$ power), 22 patients with refractory disease were to be enrolled into the study. An ORR $>5 \%$ was considered possible if at least 4 objective responses had been observed.

In sensitive disease, an ORR $\leq 15 \%$ would not have been considered of further interest. According to the Fleming's single stage design, based on our hypothesis that experimental treatment could guarantee an ORR $\geq 30 \%$ (for a $5 \%$ significance level and $80 \%$ power), 43 patients with sensitive disease were to be enrolled into the study. An ORR $>15 \%$ was considered possible if at least 11 objective responses had been observed.

The study was not designed to perform any comparison between the two cohorts.

Registered population included all patients who were enrolled into the trial. All enrolled patients who received at least one dose of nab-paclitaxel were included in the modified intention-to-treat (mITT) population and considered evaluable for activity and safety.

Descriptive tables were produced for the ORR and the best overall response. Exact binomial method was used to estimate the ORR and its $90 \%$ confidence interval.

The assessment of safety was based on the frequency of adverse events that were described as the number (and percentage) of patients reporting any adverse event, as adverse event in each body system and each individual adverse event. 


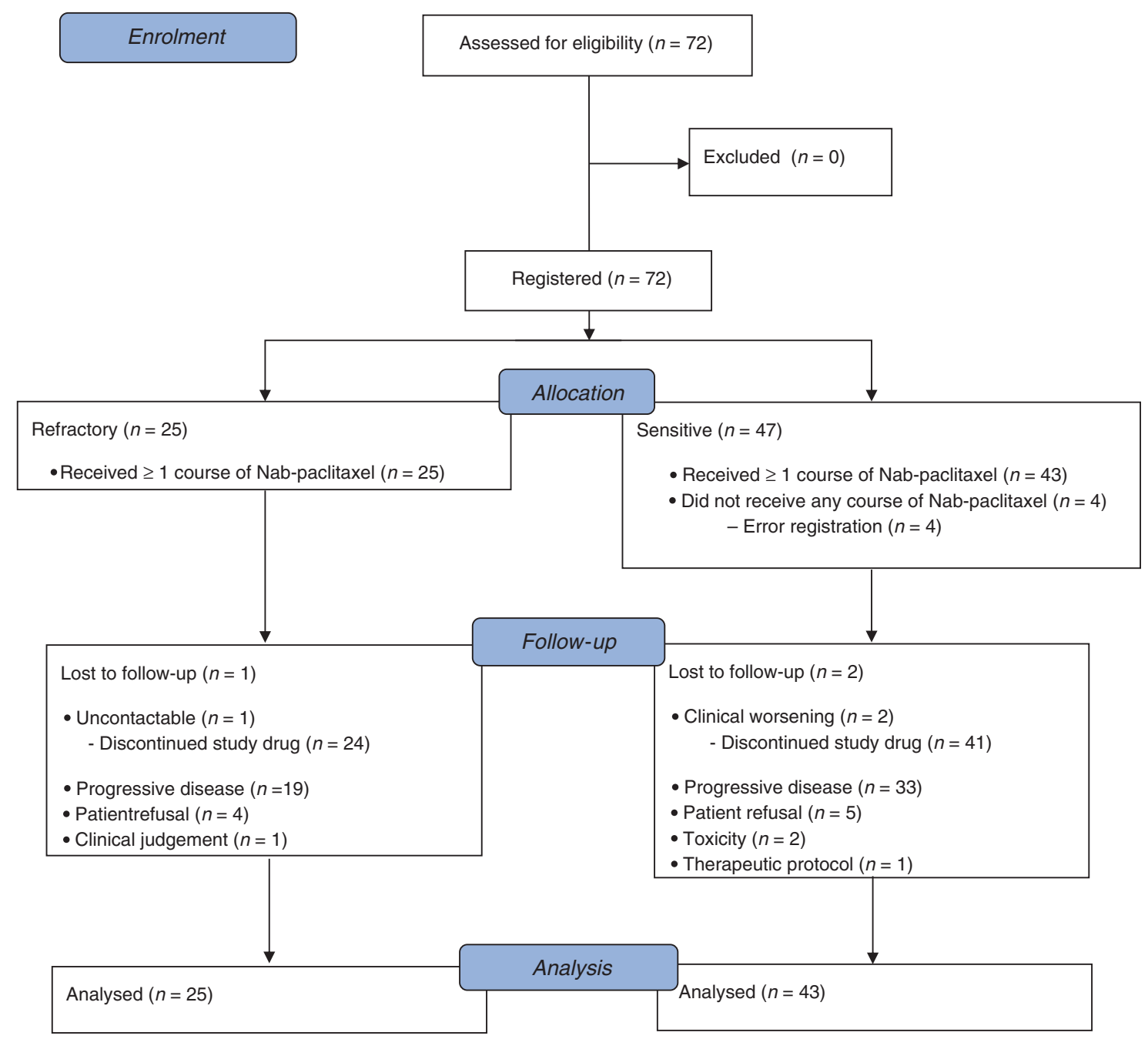

Fig. 1 CONSORT flow diagram. It displays the progress of all participants through the NABSTER trial.

Probabilities of PFS and OS were calculated according to the Kaplan-Meier product-limit method. The data cut-off for analysis was 18 October 2018.

\section{RESULTS}

Patient and treatment characteristics

Between February 2017 and March 2018, 72 patients were enrolled into the trial from 18 Italian Centres (a list of all participating Centres is available in S2, Supplemental Data). Of them, 68 patients (25 refractory and 43 sensitive) were evaluable for safety and activity and included in the mITT population (Fig. 1). Baseline patients' characteristics are shown in Table 1. With a median age of 68.5 years (44-80), a male predominance $(65 \%)$ and a high prevalence of extensive disease (84\%), our study population was quite representative of clinical practice. Notably, among patients with extensive disease, $42 \%$ had liver involvement, $12 \%$ had central nervous system (CNS) disease and $16 \%$ had both liver and brain metastases at the time of study enrolment.

The mean number of courses per patient was 2.48 in refractory group and 3.00 in sensitive one. Only $12 \%$ of patients concluded the planned treatment courses. Dose reduction occurred 61 times (32\%) in 39 patients, mainly due to haematological toxicity (26 cases). Dose delay was reported 49 times (25\%) in 33 patients. Despite dose reductions and delays, the relative dose intensity remained good $(76 \%$ in refractory cohort and $80 \%$ in sensitive cohort). All information on treatment distribution is available in S.3 (Supplemental data).

Tumour response

According to Investigator's assessment, PR was observed in 2 (8\%; IC 90\%, 1.7-24.0) patients in refractory cohort and in $6(13.9 \%$; IC 90\%, 6.6-26.1) patients in sensitive one. Thirteen (19.1\%) patients had SD, 5 patients $(20.0 \%)$ of them in refractory cohort, while 36 (52.9\%) patients had PD as best response, of whom 14 $(56.0 \%)$ in refractory group (Table 2 ).

Investigator-assessed responses were reviewed by a BIRC. According to central review assessment (Table 2), PR was observed in $4(16.0 \%$; IC $90 \%, 6.1-33.5)$ patients in refractory cohort and in 8 (18.6\%; IC 90\%, 9.9-31.4) patients in sensitive one. Eleven (16.2\%) had SD, 4 patients $(16.0 \%)$ of them in refractory cohort, while $34(50.0 \%)$ patients had PD as best response, of whom $13(52.0 \%)$ in refractory group. Finally, 11 (16.2\%) patients was not evaluated for response, 4 in refractory group and 7 in sensitive one. Waterfall plot (Fig. 2) shows the distribution and depth of response in patients evaluated for target lesions.

Notably, among $16(28 \%)$ patients with CNS involvement at baseline, 5 (31.2\%) patients obtained a brain disease control, including also 2 (22.2\%) patients with concomitant CNS and liver disease. 
Safety

All 68 patients included in the mITT population were evaluable for safety. Adverse events of any grade occurred in 53 patients (77.9\%) (Table 3). Haematological and non-haematological toxicities of any grade were reported in 36 (52.9\%) and in 49 (72.0\%) patients, respectively, whereas the same toxicities of grade 3-4 were observed in $9(13.2 \%)$ and $6(8.8 \%)$ patients, respectively. The most frequent adverse event of any grade was fatigue (54.4\%), the only

\begin{tabular}{|c|c|c|}
\hline & $\begin{array}{l}\text { Refractory } \\
(n=25)\end{array}$ & $\begin{array}{l}\text { Sensitive } \\
(n=43)\end{array}$ \\
\hline Age, median in years (range) & $65(52-80)$ & $69(44-79)$ \\
\hline \multicolumn{3}{|l|}{ Gender } \\
\hline Female & $11(44.0 \%)$ & $13(30.2 \%)$ \\
\hline Male & $14(56.0 \%)$ & $30(69.8 \%)$ \\
\hline \multicolumn{3}{|l|}{ ECOG performance status (PS) } \\
\hline 0 & $8(32.0 \%)$ & $22(51.2 \%)$ \\
\hline 1 & $17(68.0 \%)$ & $21(48.8 \%)$ \\
\hline \multicolumn{3}{|l|}{ Histology } \\
\hline SCLC & $22(88.0 \%)$ & 37 (86.0\%) \\
\hline LCNEC & $2(8.0 \%)$ & $3(7.0 \%)$ \\
\hline NEC undifferentiated & $1(4.0 \%)$ & $3(7.0 \%)$ \\
\hline \multicolumn{3}{|l|}{ Tumor stage } \\
\hline LD & $5(20.0 \%)$ & $6(14.0 \%)$ \\
\hline ED & $20(80.0 \%)$ & $37(86.0 \%)$ \\
\hline Liver & $10(50.0 \%)$ & $14(37.8 \%)$ \\
\hline Brain & $4(20.0 \%)$ & $3(8.1 \%)$ \\
\hline Liver + Brain & $3(15.0 \%)$ & $6(16.2 \%)$ \\
\hline $\begin{array}{l}\text { Treatment free interval, median } \\
\text { in days (range) }\end{array}$ & $20(0-57)$ & $123(61-820)$ \\
\hline \multicolumn{3}{|l|}{ Prior treatment } \\
\hline Chemotherapy & $24(96.0 \%)$ & $43(100 \%)$ \\
\hline Chemotherapy + ICI & $1(4.0 \%)$ & 0 \\
\hline \multicolumn{3}{|l|}{ Type of chemotherapy } \\
\hline Cisplatin + Etoposide & $4(16.0 \%)$ & $13(30.2 \%)$ \\
\hline Carboplatin + Etoposide & $21(84.0 \%)$ & $30(69.8 \%)$ \\
\hline \multicolumn{3}{|l|}{ Prior radiotherapy } \\
\hline No & 19 (76.0\%) & $13(30.2 \%)$ \\
\hline Yes & $6(24.0 \%)$ & $30(69.8 \%)$ \\
\hline
\end{tabular}

SCLC small cell lung cancer, LCNEC large cell neuroendocrine carcinoma, $L D$ limited disease, $E D$ extensive disease, $I C I$ immune checkpoint inhibitor. toxicity which led to permanent discontinuation of study drug in $2(4.6 \%)$ patients. Only one treatment-related adverse event of grade 4 (leuko-neutropenia) was reported throughout the study period. There was no treatment-related death.

\section{Survival}

The median duration of follow-up was 8.4 months (IQR, interquartile range: 5.8-12.4). Median PFS (mPFS) was 1.84 months (IC 95\%, $1.02-3.16)$ in refractory cohort, and $4.2 \%$ (IC 95\%, 0.3-17.7) of these patients were free from disease progression at 6 months (Fig. 3a). Similar results were observed in sensitive group, for which mPFS was 1.88 months (IC 95\%, 1.81-2.37), with a 6-month PFS rate of $10.1 \%$ (IC 95\%, 3.2-21.5) (Fig. 3a).

Median OS (mOS) was 3.65 months (IC 95\%, 2.07-4.57) in refractory cohort and 20.9\% (IC 95\%, 7.6-38.6) of these patients were alive at 6 months (Fig. 3b), whereas in sensitive cohort mOS was 6.64 months (IC 95\%, 3.16-9.70), with a 6-month OS rate of $60.8 \%$ (IC 95\%, 44.1-73.9) (Fig. 3b). At the time of data cut-off, no patient was still being treated, although 4 (5.9\%) patients ( 1 in refractory cohort and 3 patients in sensitive one) had no event, and $58(85.3 \%)$ patients progressed, of whom $22(88.0 \%)$ were refractory. Twenty-one (30.9\%) patients were alive (3 refractory and 18 sensitive), while 47 (69.1\%) patients were dead, 40 (58.8\%) of them due to disease progression (19 and 21 patients in refractory and sensitive cohorts, respectively).

\section{DISCUSSION}

Based on its poor prognosis and survival plateau achieved in the last decades, SCLC has been defined one of the recalcitrant cancers. Till now, several treatment strategies and clinical trial designs have been developed with daunting results. Therefore, there is an urgent need for additional and effective therapeutic innovations. The impressive results of immune checkpoint inhibitors, such as the monoclonal antibodies directed against programmed cell death-1 (PD-1) and its ligand (PD-L1), for the treatment of different solid tumours, have led to evaluate them also in SCLC. In the last year, two randomised, controlled Phase 3 trials showed that adding atezolizumab (IMpower-133 study) or durvalumab (CASPIAN study), two antibodies directed against PD-L1, to standard first-line chemotherapy led to a statistically significant improvement in terms of OS in patients with EDSCLC, although this benefit would not be considered clinically significant. $^{23,24}$

In second-line setting, different clinical studies are investigating the efficacy of several new agents, either alone or combined to standard chemotherapy.

Our study is the first prospective trial of nab-paclitaxel for relapsed SCLC. Overall, this trial showed a modest anti-cancer activity, so that it did not meet its primary endpoint (ORR), in both refractory and sensitive cohorts. Based on investigator's

Table 2. Best overall response based on both Investigator and BIRC assessment.

\begin{tabular}{|c|c|c|c|c|c|c|}
\hline CR & 0 & 0 & 0 & 0 & 0 & 0 \\
\hline PR & $2(8.0)$ & 6 (13.9) & 8 (11.8) & $4(16.0)$ & 8 (18.6) & 12 (17.6) \\
\hline SD & $5(20.0)$ & 8 (18.6) & $13(19.1)$ & $4(16.0)$ & 7 (16.3) & $11(16.2)$ \\
\hline PD & $14(56.0)$ & $22(51.2)$ & 36 (52.9) & $13(52.0)$ & $21(41.8)$ & $34(50.0)$ \\
\hline
\end{tabular}




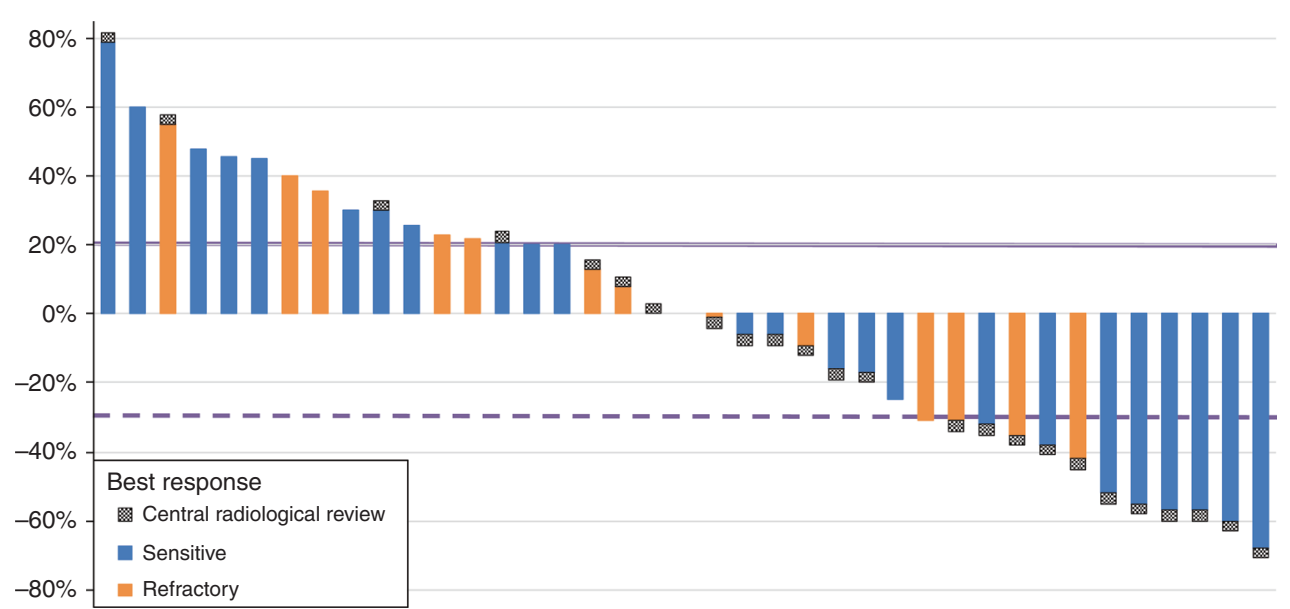

Fig. 2 Waterfall plot. It describes the changes in tumor size in all evaluable participants with target lesions. Blue bars represent sensitive patients, while orange bars are refractory ones. The black/white signal at the top of each bar corresponds to central radiological review for each individual patient.

Table 3. Toxicity profile.

\begin{tabular}{lll}
\hline & Any grade & Grade $\geq 3$ \\
\hline Fatigue & $37(54.4 \%)$ & $3(4.4 \%)$ \\
Anemia & $25(36.7 \%)$ & $1(1.4 \%)$ \\
Neutropenia & $20(29.4 \%)$ & $7(10.3 \%)$ \\
Leukopenia & $18(26.4 \%)$ & $3(4.4 \%)$ \\
Diarrhea & $14(20.5 \%)$ & 0 \\
Nausea & $13(19.1 \%)$ & 0 \\
Peripheral neuropathy & $13(19.1 \%)$ & 0 \\
Fever without neutropenia & $10(14.7 \%)$ & 0 \\
Vomiting & $8(11.7 \%)$ & $1(1.4 \%)$ \\
Trombocytopenia & $7(10.3 \%)$ & 0 \\
Constipation & $6(8.8 \%)$ & 0 \\
Skin toxicity & $5(7.3 \%)$ & $1(1.4 \%)$ \\
Mucositis & $5(7.3 \%)$ & 0 \\
Liver toxicity & $3(4.4 \%)$ & $1(1.4 \%)$ \\
Renal toxicity & $1(1.4 \%)$ & 0 \\
\hline
\end{tabular}

assessment and study design, there were 2 tumour responses $(\mathrm{ORR}, 8 \%)$ out of 4 or more required in refractory group and 6 tumour responses (ORR, 13.9\%) out of 11 or more required in sensitive one, needed to reach the primary objective of the study. However, after central independent radiological review, two additional cases of objective response were identified in the refractory group which would qualify the study as positive, at least in this cohort. Secondary endpoints of the study included PFS, OS and toxicity. Data on survival outcomes confirmed the dismal prognosis of these patients, with a mPFS less than 2 months in both refractory and sensitive cohorts and a mOS that was almost double in sensitive group (6.64 months) compared to refractory one (3.65 months). Furthermore, although nearly $30 \%$ of patients with CNS involvement at baseline had a brain disease control, 11 $(68.7 \%)$ out of 16 patients experienced a rapid progressive disease (within 1-2 courses), including two patients with early death. These data confirmed the unfavourable prognostic role of CNS involvement, especially in relapsed SCLC.

Our results were similar to those reported from a retrospective study ${ }^{18}$ in which 9 of the 14 enrolled patients were treated with nab-paclitaxel, as third-line or later. In this subgroup, ORR, mPFS and $\mathrm{mOS}$ were $11 \%, 2.0$ months and 4.0 months, respectively.
Almost all patients were refractory to first-line chemotherapy regimen, but the authors did not report any information on the prevalence of brain and liver metastases in this population.

Similarly, a retrospective analysis reported outcome of 31 heavily pre-treated Japanese SCLC patients of whom only 4 received nab-paclitaxel, preventing any meaningful consideration on the efficacy of this agent. ${ }^{20}$

In our study, the discordance in terms of ORR between local and central assessment has been mainly due, at least in some cases, to an improper application of RECIST v1.1 by local radiologists. For example, two refractory patients considered as stable were reclassified as responders after central radiological review because of a misleading interpretation of two target liver lesions in one case and two pathological mediastinal lymph nodes in the other one. These results in refractory cohort are not similar to those reported from different Phase 2 trials that showed how paclitaxel had a promising anti-tumour activity, reaching a response rate of $20-29 \% .{ }^{9,25}$ A higher response rate $(41 \%)$ was reported from a Phase 2 trial of irinotecan administered in 30 Japanese patients with relapsed SCLC. However, it is reasonable to believe that patient population included into this study was "positively" selected. In fact, all patients had ECOG PS 0 or 1 , one third of them had LD-stage, $60 \%$ had sensitive recurrent disease, with only $10 \%$ and $13 \%$ of patients having brain and liver involvement, respectively. ${ }^{26}$ Similar results were reported from a multicentre, single-arm Phase 2 basket study of lurbinectedin, a RNA polymerase II inhibitor, in patients across advanced solid tumours. Thirty-seven (35.2\%) out of 105 enrolled SCLC patients had a partial response. Overall, median PFS and OS times were 3.9 months $(95 \% \mathrm{Cl}, 2.6-4.6)$ and 9.3 months $(95 \% \mathrm{Cl}, 6.3-11.8)$. According to TFI (<or $\geq 90$ days), these clinical outcomes have more than doubled in sensitive patients $(45 \%, 4.6$ months and 11.9 months) compared to refractory ones $(22.2 \%, 2.6$ months and 5.0 months). Lurbinectedin showed a favourable and manageable toxicity profile. The most common grade $1-2$ adverse events were fatigue $(51.4 \%)$, nausea $(32.4 \%)$, decreased appetite $(21 \%)$, vomiting (18.1\%) and diarrhoea (12.4\%). Grade 3-4 adverse events included neutropenia (22.9\%), anaemia and fatigue $(6.7 \%$ each), febrile neutropenia and thrombocytopenia (4.8\% each). ${ }^{27}$

To date, topotecan remains the only drug approved for relapsed SCLC patients, based on the results of different Phase 2-3 trials that showed a response rate of $7-38 \%$ among sensitive patients and of $2-7 \%$ among refractory ones. ${ }^{5-7,28,29}$ A recent meta-analysis described clinical outcomes of 1347 SCLC patients treated with topotecan from 14 prospective trials. ${ }^{30}$ Objective tumour response and 6 -month OS rates were $5 \%$ and $37 \%$ in refractory patients and 

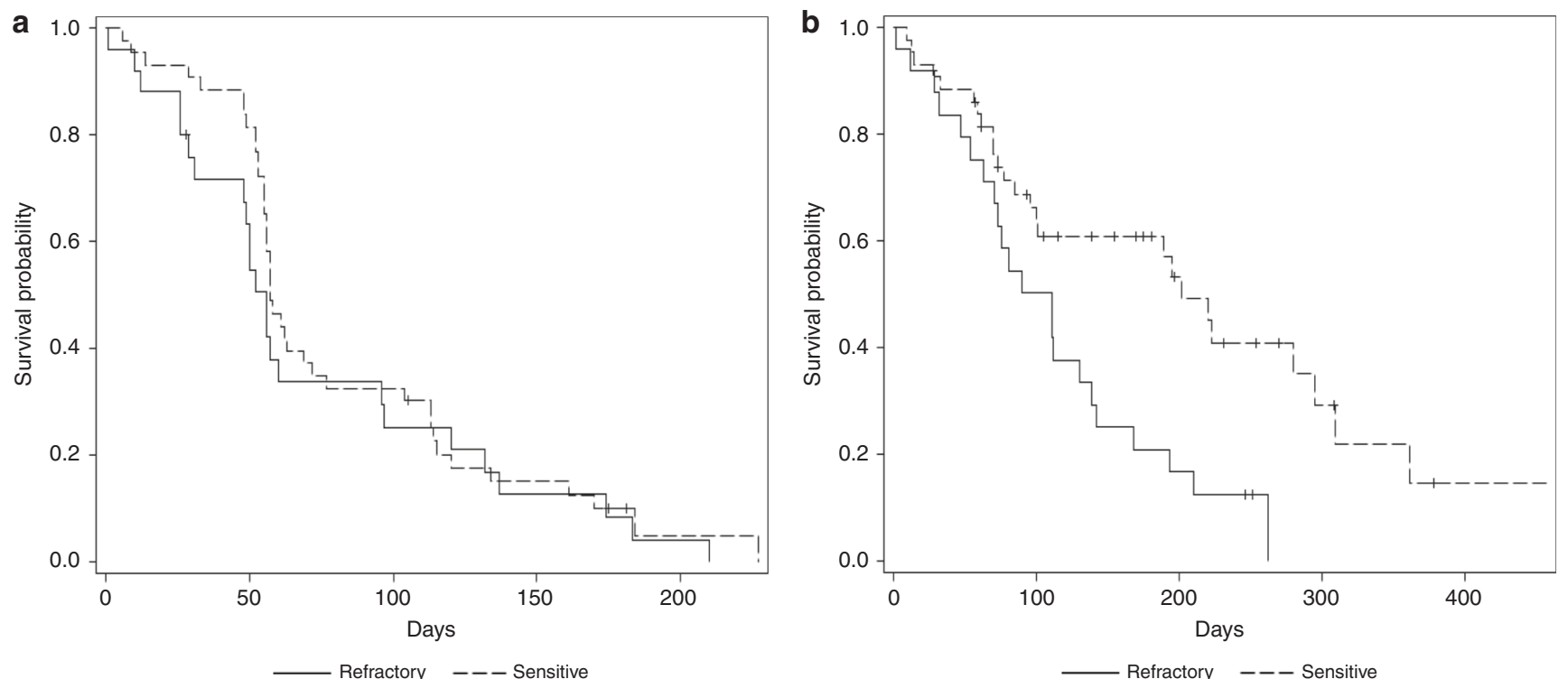

Fig. 3 PFS and OS in modified ITT population. Probabilities of PFS (a) and OS (b) were calculated according to the Kaplan-Meier product-limit method. In both graphs (a and $\mathbf{b})$, continuous and dashed curves represent survival probabilities in refractory and sensitive cohorts, respectively.

$17 \%$ and $57 \%$ in sensitive ones, respectively. Notably, these data are in line with the results of our study. Results from clinical studies investigating the role of anti-PD-1/PD-L1 drugs in secondor further-line setting were conflicting so far, particularly when used as single-agent. ${ }^{31-36}$ In the recently reported Phase 3 CheckMate-331 trial of nivolumab, a human lgG4 monoclonal antibody against PD-1, 569 SCLC patients relapsed on or following platinum-based chemotherapy were randomised (1:1) to receive either nivolumab $(N=284)$ or standard second-line chemotherapy (topotecan or amrubicin, $N=285) .{ }^{37}$ Results of this study showed that, after 7.0-7.6 months of median follow-up, nivolumab did not yield a significant survival improvement (primary endpoint) compared to the standard chemotherapy arm (7.5 months [95\% $\mathrm{Cl}$ 5.6-9.2] versus 8.4 months [95\% $\mathrm{Cl} 7.0-10.0], p=0.11)$. This confirms that, at least in a subset of relapsed SCLC patients, chemotherapy is the option of choice.

Based on safety, nab-paclitaxel has shown a favourable toxicity profile, particularly considering historical data on topotecan. Nabpaclitaxel was well tolerated, and the proposed schedule was feasible. The most common grade 3-4 adverse events were neutropenia (10\%), leukopenia and fatigue (4\% each) and anaemia (1\%). Conversely, topotecan was encumbered with a high incidence of severe (grade 3-4) haematological toxicity, including neutropenia (69\%), thrombocytopenia (41\%) and anaemia (24\%). ${ }^{30}$

Although nab-paclitaxel has demonstrated a not negligible antitumour activity, particularly in refractory relapsed SCLC, associated with a favourable toxicity profile, the primary end-point of the study was not meet. Based on these results, we believe that further studies comparing nab-paclitaxel to the current standardof-care topotecan would not be justified.

\section{ACKNOWLEDGEMENTS}

We thank all Centres, Investigators and patients for participating to the study and Dr. Michele Tognetto, Laura Casolari and Daniela Baldari for their technical support.

\section{AUTHOR CONTRIBUTIONS}

F.G., M.T., G.L., L.B. and A.A. wrote full protocol and defined study design. A.A. obtained funding for the study. F.G., M.T., F.B., F.R., L.C., A.F., A.D., L.L., C.D., S.C., I.C., F.S. and A.A. enrolled patients onto the study. S.B. reviewed in blinded all available imaging scans. F.G., L.T., L.B. and A.A. collected and prepared data for the analysis. L.T. and L.B. conducted the analyses. F.G., M.T., L.B. and A.A. wrote and revised full paper. All authors read and approved the revised and final versions of the paper.

\section{ADDITIONAL INFORMATION}

Ethics approval and consent to participate The study protocol was approved by each local institutional ethics committee (a list of the Ethics Committees that approved the study is available in S.4, Supplemental Data) and conducted in accordance with the ICH Harmonized Tripartite Guidelines for Good Clinical Practice and the Declaration of Helsinki. Written informed consent was obtained from all participants.

Consent to publish No individually identifiable data is presented.

Data availability Anonymised dataset may be available from the corresponding author on reasonable request.

Competing interests A.A. has received research grant support from BMS and Celgene; personal fees for serving in a consultant and/or advisory role for BMS, MSD and Boehringer; honoraria from Eli-Lilly and Pfizer. A.F. has received personal fees for serving in a consultant and/or advisory role for Novartis, Roche and Astrazeneca; honoraria from Novartis, Astrazeneca, Pfizer, Lilly, Eisai, Roche, Novartis and Celgene. L.C. has received personal fees for serving in a consultant and/or advisory role for Astrazeneca and Merck; honoraria from Celgene, Pfizer and Ipsen. The remaining authors declare no competing interests.

Funding information The study was sponsored by Gruppo Oncologico Italiano di Ricerca Clinica (GOIRC) and partially supported by Celgene that provided investigational medicinal product and a restricted grant for the management of study procedures.

Supplementary information is available for this paper at https://doi.org/10.1038/ s41416-020-0845-3.

Publisher's note Springer Nature remains neutral with regard to jurisdictional claims in published maps and institutional affiliations.

\section{REFERENCES}

1. van Meerbeeck, J. P., Fennell, D. A. \& De Ruysscher, D. K. Small-cell lung cancer. Lancet 378, 1741-1755 (2011).

2. Govindan, R., Page, N., Morgensztern, D., Read, W., Tierney, R., Vlahiotis, A. et al Changing epidemiology of small-cell lung cancer in the United States over the 
last 30 years: analysis of the surveillance, epidemiologic, and end results database. J. Clin. Oncol. 24, 4539-4544 (2006).

3. Albain, K. S., Crowley, J. J., LeBlanc, M. \& Livingston, R. B. Determinants of improved outcome in small-cell lung cancer: an analysis of the 2,580-patient Southwest Oncology Group data base. J. Clin. Oncol. 8, 1563-1574 (1990).

4. Ardizzoni, A., Tiseo, M. \& Boni, L. Validation of standard definition of sensitive versus refractory relapsed small cell lung cancer: a pooled analysis of topotecan second-line trials. Eur. J. Cancer 50, 2211-2218 (2014).

5. O'Brien, M. E., Ciuleanu, T. E., Tsekov, H., Shparyk, Y., Cuceviá, B., Juhasz, G. et al. Phase III trial comparing supportive care alone with supportive care with oral topotecan in patients with relapsed small-cell lung cancer. J. Clin. Oncol. 24, 5441-5447 (2006).

6. Ardizzoni, A., Hansen, H., Dombernowsky, P., Gamucci, T., Kaplan, S., Postmus, P. et al. Topotecan, a new active drug in the second-line treatment of small-cell lung cancer: a phase II study in patients with refractory and sensitive disease. The European Organization for Research and Treatment of Cancer Early Clinical Studies Group and New Drug Development Office, and the Lung Cancer Cooperative Group. J. Clin. Oncol. 15, 2090-2096 (1997).

7. von Pawel, J., Schiller, J. H., Shepherd, F. A., Fields, S. Z., Kleisbauer, J. P., Chrysson, N. G. et al. Topotecan versus cyclophosphamide, doxorubicin, and vincristine for the treatment of recurrent small-cell lung cancer. J. Clin. Oncol. 17, 658-667 (1999)

8. Ardizzoni, A. Topotecan in the treatment of recurrent small cell lung cancer: an update. Oncologist 9, 4-13 (2004).

9. Smit, E. F., Fokkema, E., Biesma, B., Groen, H. J., Snoek, W. \& Postmus, P. E. A phase II study of paclitaxel in heavily pretreated patients with small-cell lung cancer. $B r$. J. Cancer 77, 347-351 (1998).

10. Groen, H. J., Fokkema, E., Biesma, B., Kwa, B., van Putten, J. W., Postmus, P. E. et al. Paclitaxel and carboplatin in the treatment of small-cell lung cancer patients resistant to cyclophosphamide, doxorubicin, and etoposide: a non-cross-resistant schedule. J. Clin. Oncol. 17, 927-932 (1999).

11. de Jong, W. K., Groen, H. J., Koolen, M. G., Biesma, B., Willems, L. N., Kwa, H. B. et al. Phase III study of cyclophosphamide, doxorubicin, and etoposide compared with carboplatin and paclitaxel in patients with extensive disease small-cell lung cancer. Eur. J. Cancer 43, 2345-2350 (2007).

12. Ibrahim, N. K., Desai, N., Legha, S., Soon-Shiong, P., Theriault, R. L., Rivera, E. et al. Phase I and pharmacokinetic study of ABI-007, a Cremophor-free, protein-stabilized, nanoparticle formulation of paclitaxel. Clin. Cancer Res. 8, 1038-1044 (2002).

13. Desai, N., Trieu, V., Yao, Z., Louie, L., Ci, S., Yang, A. et al. Increased antitumor activity, intratumor paclitaxel concentrations, and endothelial cell transport of cremophor-free, albumin-bound paclitaxel, ABI-007, compared with cremophorbased paclitaxel. Clin. Cancer Res. 12, 1317-1324 (2006).

14. Yardley, D. A. nab-Paclitaxel mechanisms of action and delivery. J. Control Release 170, 365-372 (2013)

15. Gradishar, W. J., Tjulandin, S., Davidson, N., Shaw, H., Desai, N., Bhar, P. et al. Phase III trial of nanoparticle albumin-bound paclitaxel compared with polyethylated castor oil-based paclitaxel in women with breast cancer. J. Clin. Oncol. 23, 7794-7803 (2005).

16. Von Hoff, D. D., Ervin, T., Arena, F. P., Chiorean, E. G., Infante, J., Moore, M. et al. Increased survival in pancreatic cancer with nab-paclitaxel plus gemcitabine. $N$. Engl. J. Med. 369, 1691-1703 (2013).

17. Socinski, M. A., Bondarenko, I., Karaseva, N. A., Makhson, A. M., Vynnychenko, l., Okamoto, I. et al. Weekly nab-paclitaxel in combination with carboplatin versus solvent-based paclitaxel plus carboplatin as first-line therapy in patients with advanced non-small-cell lung cancer: final results of a phase III trial. J. Clin. Oncol. 30, 2055-2062 (2012).

18. Naito, Y., Tamiya, A., Tamiya, M., Kimura, Y., Hamaguchi, M., Saijo, N. et al. Efficacy of nanoparticle albumin-bound paclitaxel regimens for relapsed small cell lung cancer: a retrospective analysis. Medicine 96, e7884 (2017).

19. Yoshida, H., Kim, Y. H., Ozasa, H., Nagai, H., Sakamori, Y., Nakaoku, T. et al. Albumin-bound paclitaxel for the treatment of refractory or relapsed small-cell lung cancer. Mol. Clin. Oncol. 5, 213-215 (2016).

20. Sugiyama, K., Kogure, Y., Torii, A., Shiraishi, K., Yamada, A., Ishida, A. et al. Solventbased paclitaxel or nab-paclitaxel for heavily treated relapsed/refractory small cell lung cancer: retrospective single-institution observational study. Medicine $\mathbf{9 8 ,}$ e14758 (2019).

21. Travis W. D., Brambilla E., Burke A. P., Marx A., Nicholson A. G. WHO Classification of Tumours of the Lung, Pleura, Thymus and Heart. (International Agency for Research on Cancer, Lyon, 2015)
22. Eisenhauer, E. A., Therasse, P., Bogaerts, J., Schwartz, L. H., Sargent, D., Ford, R. et al. New response evaluation criteria in solid tumours: revised RECIST guideline (version 1.1). Eur. J. Cancer 45, 228-247 (2009).

23. Horn, L., Mansfield, A. S., Szczęsna, A., Havel, L., Krzakowski, M., Hochmair, M. J. et al. First-Line Atezolizumab plus chemotherapy in extensive-stage small-cell lung cancer. N. Engl. J. Med. 379, 2220-2229 (2018).

24. Paz-Ares, L., Dvorkin, M., Chen, Y., Reinmuth, N., Hotta, K., Trukhin, D. et al. Durvalumab plus platinum-etoposide versus platinum-etoposide in first-line treatment of extensive-stage small-cell lung cancer (CASPIAN): a randomised, controlled, open-label, phase 3 trial. Lancet 394, 1929-1939 (2019).

25. Joos G., Schallier D., Pinson P., et al. Paclitaxel (PTX) as second line treatment in patients (pts) with small cell lung cancer (SCLC) refractory to carboplatin-etoposide: a multicenter phase II study. Proc. Am. Soc. Clin. Oncol. 22, abstr. 7211 (2004).

26. Kondo, R., Watanabe, S., Shoji, S., Ichikawa, K., Abe, T., Baba, J. et al. A phase II study of irinotecan for patients with previously treated small-cell lung cancer. Oncology 94, 223-232 (2018).

27. Paz-Ares L. G., Perez J. M. T., Besse B., Moreno V., Lopez R., Sala M. A., et al. Efficacy and safety profile of lurbinectedin in second-line SCLC patients: Results from a phase II single-agent trial. J. Clin. Oncol. 37, abstr. 8506 (2019).

28. von Pawel, J., Gatzemeier, U., Pujol, J. L., Moreau, L., Bildat, S., Ranson, M. et al. Phase II comparator study of oral versus intravenous topotecan in patients with chemosensitive small-cell lung cancer. J. Clin. Oncol. 19, 1743-1749 (2001).

29. Eckardt, J. R., von Pawel, J., Pujol, J. L., Papai, Z., Quoix, E., Ardizzoni, A. et al. Phase III study of oral compared with intravenous topotecan as second-line therapy in small-cell lung cancer. J. Clin. Oncol. 25, 2086-2092 (2007).

30. Horita, N., Yamamoto, M., Sato, T., Tsukahara, T., Nagakura, H., Tashiro, K. et al. Topotecan for relapsed small-cell lung cancer: systematic review and metaanalysis of 1347 patients. Sci. Rep. 5, 15437 (2015).

31. Antonia, S. J., López-Martin, J. A., Bendell, J., Ott, P. A., Taylor, M., Eder, J. P. et al. Nivolumab alone and Nivolumab plus ipilimumab in recurrent small-cell lung cancer (CheckMate 032): a multicentre, open-label, phase 1/2 trial. Lancet Oncol. 17, 883-895 (2016).

32. Ott, P., Felip, E., Hiret, S., Kim, D.-W., Morosky, A., Saraf, S. et al. Pembrolizumab in patients with extensive-stage small cell lung cancer: updated survival results from KEYNOTE-028. J. Thorac. Oncol. 12(Suppl. 1), S259 (2017). abstract OA05.01.

33. Chung H. C., Lopez-Martin J. A., Kao S. C.-H., Miller W. H., Ros W., Gao B., et al. Phase 2 study of embrolizumab in advanced small-cell lung cancer (SCLC): KEYNOTE-158. J. Clin. Oncol. 36 (Suppl. 15), abstr. 8506 (2018)

34. Goldman J. W., Dowlati A., Antonia S. J., Nemunaitis J. J., Butler M. O., Segal N. H., et al. Safety and antitumor activity of durvalumab monotherapy in patients with pretreated extensive disease small-cell lung cancer (ED-SCLC). J. Clin. Oncol. 36 (Suppl. 15), abstr. 8518 (2018).

35. Hellmann M. D., Ott P. A., Zugazagoitia J., Ready N. E., Hann C. L., De Braud F. G., et al. Nivolumab (nivo) \pm ipilimumab (ipi) in advanced small-cell lung cancer (SCLC): First report of a randomized expansion cohort from CheckMate 032. J. Clin. Oncol. 35 (Suppl. 15), abstr. 8503 (2017)

36. Ready, N., Farago, A. F., de Braud, F., Atmaca, A., Hellmann, M. D., Schneider, J. G. et al. Third-line nivolumab monotherapy in recurrent small cell lung cancer: CheckMate 032. J. Thorac. Oncol. 14, 237-244 (2019).

37. Reck, M., Vicente, D., Ciuleanu, T., Gettinger, S., Peters, S., Horn, L. et al. Efficacy and safety of nivolumab (nivo) monotherapy versus chemotherapy (chemo) in recurrent small cell lung cancer (SCLC): results from CheckMate 331. Ann. Oncol. 29(Suppl. 10), x39-x43 (2018).

Open Access This article is licensed under a Creative Commons Attribution 4.0 International License, which permits use, sharing, adaptation, distribution and reproduction in any medium or format, as long as you give appropriate credit to the original author(s) and the source, provide a link to the Creative Commons license, and indicate if changes were made. The images or other third party material in this article are included in the article's Creative Commons license, unless indicated otherwise in a credit line to the material. If material is not included in the article's Creative Commons license and your intended use is not permitted by statutory regulation or exceeds the permitted use, you will need to obtain permission directly from the copyright holder. To view a copy of this license, visit http://creativecommons. org/licenses/by/4.0/.

(c) The Author(s) 2020, corrected publication 2021 